\title{
PORTFOLIO OPTIMIZATION WITH JUMPS AND UNOBSERVABLE INTENSITY PROCESS
}

\author{
NiCOLE BÄUERLE \\ Institute for Mathematical Stochastics, University of Karlsruhe, Germany \\ ULRICH RIEDER \\ Department of Optimization and Operations Research, University of Ulm, Germany
}

We consider a financial market with one bond and one stock. The dynamics of the stock price process allow jumps which occur according to a Markov-modulated Poisson process. We assume that there is an investor who is only able to observe the stock price process and not the driving Markov chain. The investor's aim is to maximize the expected utility of terminal wealth. Using a classical result from filter theory it is possible to reduce this problem with partial observation to one with complete observation. With the help of a generalized HamiltonJacobi-Bellman equation where we replace the derivative by Clarke's generalized gradient, we identify an optimal portfolio strategy. Finally, we discuss some special cases of this model and prove several properties of the optimal portfolio strategy. In particular we derive bounds and discuss the influence of uncertainty on the optimal portfolio strategy.

KEY WORDS: jump-diffusion process, filtering, utility maximization, stochastic control, generalized HJB equation, optimal portfolio strategies, Bayesian control, stochastic comparison

\section{Introduction}

We consider an incomplete financial market with one bond and one stock. The stock price process allows for jumps of a random height where the jump time points are generated by a Markov-modulated Poisson process. There is an investor who wants to maximize his utility from terminal wealth and who is only able to observe the stock price process. In particular he is not informed about the state of the continuous-time Markov chain which drives the jump intensity. Such a model is also called a Hidden Markov Model. For a general treatment of such models see e.g. Elliott et al. (1994).

A model with unobservable intensity process is natural, since jumps in the stock price process are often generated by various external events whose impact on the stock market

\footnotetext{
${ }^{0}$ Acknowledgement: We are grateful to an anonymous referee for careful reading. Address correspondence to Nicole Bäuerle, Institute for Mathematical Stochastics, University of Karlsruhe, D-76128 Karlsruhe, Germany, e-mail: baeuerle@stoch.uni-karlsruhe.de
} 
cannot completely be analyzed. We are only able to draw some conclusions about the jump intensity from the observation of the stock prices. Also it is more appropriate to allow a stochastically varying jump intensity since a deterministic jump intensity seems only to be realistic for a short period of time. A continuous-time Markov chain can model the changing conditions which give rise to a changing jump behavior. This underlying Markov chain can be interpreted as an environment process which collects factors which are relevant for the stock price dynamics like technical progress, political situations, law or natural catastrophes.

There is an extensive literature on portfolio optimization with partial observation as well as on portfolio optimization with discontinuous stock price processes. In this paper we will treat these two aspects in one model.

Most papers on problems with partial observation deal with the case of an unobserved (stochastic) appreciation rate process $\left(\mu_{t}\right)$. Lakner $(1995,1998)$ for example treats the case where the appreciation rate follows a linear Gaussian model. The most recent papers by Honda (2003), Sass and Haussmann (2004), Haussmann and Sass (2004) and Rieder and Bäuerle (2005) consider a Hidden Markov Model for $\left(\mu_{t}\right)$. We refer the reader to these papers for a recent survey on financial models with partial observation. Of course it would be more realistic to assume that both the appreciation rate and the jump intensity depend on the hidden Markov chain but this seems to be too challenging at the moment. For a risk averse investor it might be more important to model an unobserved jump intensity than an unobserved appreciation rate, since potential losses due to jumps can be much higher. In order to solve these problems the usual technique is to use the well-established filter theory to reduce the stochastic control problem with partial observation to one with complete observation. It is then possible to solve this problem either with stochastic control methods or via the martingale approach (which is mostly done in case of a complete market in the literature).

On the other hand there exist several papers on portfolio optimization problems with discontinuous stock price processes, in particular in the case where the price is modelled with the help of a Lévy process. Empirical work has shown that logreturns are in general not normally distributed and that stock price models should contain a jump component. In Framstad et al. (1999), the authors deal with the problem of optimal consumption and portfolio selection in a model where the stock price follows a geometric Lévy process. They assume a power utility and solve the problem explicitly by showing that the value function is a classical solution of the associated Hamilton-Jacobi-Bellman equation. Benth et al. (2001) consider a similar question in the case that the stock price is given by an exponential Lévy process. They have to use the notion of a constrained viscosity solution to characterize their solution. As we will see later, our price process cannot be written as a functional of a Lévy process. Important applications of optimization problems with jumps are well-known in risk theory and insurance mathematics (see e.g. Hipp and Plum (2003), Schmidli (2002)). But in these papers the intensity process is always observable.

In this paper we combine the jump diffusion model with an unknown jump intensity. Our main contributions are a non-standard approach to solve the stochastic control problem by a generalized Hamilton-Jacobi-Bellman (HJB) equation which might be interesting for other portfolio problems as well and moreover, a study of the influence of uncertainty on the optimal portfolio strategy. The outline of the paper is as follows. In Section 2 we give a precise mathematical formulation of our model and define the optimization problem. In Section 3 we show how we can use filtering theory to reduce the problem to one with complete observation. The reduced market model we end up is not complete. Thus, the martingale approach cannot be 
applied directly. In the case of a logarithmic utility function, it is shown in section 4 that the portfolio optimization problem can be solved (as usual) rather easily by a pathwise optimization. Section 5 deals with the power utility. Here we use the theory of stochastic control to solve the problem explicitly. The value function is not a classical solution of the corresponding Hamilton-Jacobi-Bellman equation, however, we can characterize the value function as a solution of a generalized HJB equation where we use the Clarke generalized gradient. This is possible since the value function can be shown to be locally Lipschitz-continuous and almost convex. This approach is non-standard and has the advantage that the optimal portfolio strategy can be given rather explicitly. Most interesting is the fact that the expression for the optimal portfolio strategy includes the value function but no derivative of it. Section 5.1 contains the main results. In Section 5.2 we deal with some special cases and derive some important properties and comparison results for the optimal portfolio strategy. In particular we highlight the role of uncertainty in our model. It turns out that adding more uncertainty by jumps reduces the investment in the stock for all investors with power utility. Moreover, we look at the Bayesian model, i.e. when the jump intensity is constant but unobservable. We are able to derive bounds and compare the optimal portfolio strategy in this case with the strategy we obtain in a model where the constant jump intensity is equal to the estimated one. The comparison depends on whether jumps go upwards or downwards and on the parameter of the power utility function. In this case further uncertainty does not automatically lead to a smaller investment in the stock. Some auxiliary results which are needed for the proof of our main theorems are given in Section 6. Section 7 finally contains the proofs of our main theorems in the case of a power utility.

\section{The Model}

We consider a financial market with one bond and one risky asset. More precisely let $(\Omega, \mathcal{F}, \mathfrak{F}=$ $\left.\left\{\mathcal{F}_{t}, 0 \leq t \leq T\right\}, P\right)$ be a filtered probability space. $T>0$ is a fixed time horizon. The bond price process $\left(B_{t}\right)$ evolves according to

$$
d B_{t}=r B_{t} d t
$$

with interest rate $r>0$ and the stock price process evolves according to

$$
d S_{t}=S_{t-}\left(\mu d t+\sigma d W_{t}+\int y N(d t, d y)\right),
$$

where $\sigma>0, \mu \in \mathbb{R}$ are given constants and $\left(W_{t}\right)$ is a Brownian motion w.r.t. $\mathfrak{F}$. $N$ is the random counting measure of a Markov-modulated compound Poisson process. That means $N$ is constructed as follows: let us denote by $\left(Y_{t}\right)$ a continuous-time Markov chain with state space $\left\{e_{1}, \ldots, e_{d}\right\}$ where $e_{k}$ is the $k$-th unit vector in $\mathbb{R}^{d}$ and $\left(Y_{t}\right)$ has the generator $Q_{0}=\left(q_{i j}\right) . q_{i j}$ is the intensity of getting from state $e_{i}$ in state $e_{j}$. Further let us denote by $T_{0}=0, T_{1}, T_{2}, \ldots$ the jump time points of a Poisson process with $\mathfrak{F}$-intensity $\left(\lambda_{t}\right):=\left(\lambda^{\prime} Y_{t}\right)$ where $\lambda=\left(\lambda_{1}, \ldots, \lambda_{d}\right) \in$ $\mathbb{R}_{+}^{d}$, i.e. as long as $Y_{t}=e_{j}$, jumps arrive at rate $\lambda_{j}$. Finally we assume that $\left(\delta_{n}\right)$ is a sequence of independent and identically distributed random variables bounded from above with $\delta_{n}>-1$. The probability distribution of $\delta_{n}$ is denoted by $Q$. Then we have

$$
N=\sum_{n \geq 1} \boldsymbol{\epsilon}_{\left(T_{n}, \delta_{n}\right)}
$$


where $\boldsymbol{\epsilon}_{x}$ is the one point measure in $x . \delta_{n}$ is the relative jump height of the stock at time $T_{n}$. Note that the restriction $\delta_{n}>-1$ guarantees that the stock price stays positive. The compound Poisson process which describes the jumps is then obtained by

$$
\int_{0}^{t} \int y N(d s, d y)=\sum_{n=1}^{N_{t}} \delta_{n}
$$

The economic interpretation of $\left(Y_{t}\right)$ is some kind of environment process which collects factors which are relevant for the stock price dynamics like e.g. technical progress, political situations, laws or natural catastrophes. These factors change stochastically over time. All processes are adapted w.r.t. $\mathfrak{F}$ and $\left(W_{t}\right)$ and $N$ are independent as well as $\left(\delta_{n}\right)$ and $\left(Y_{t}\right)$.

In what follows we assume an investor who is only able to observe the stock price process and who knows the distribution of $Y_{0}$. This means that the investor is not informed about the intensity with which the stock price process jumps. Of course it is more realistic to assume that the appreciation rate of the stock price also depends on the unobservable environment process $\left(Y_{t}\right)$, however this case is much more challenging. The case of unobservable Markovmodulated appreciation rate has been investigated in Honda (2003), Sass and Haussmann (2004) , Haussmann and Sass (2004) and Rieder and Bäuerle (2005) among others.

Let $\mathfrak{F}^{S}=\left(\mathcal{F}_{t}^{S}\right)$ be the filtration generated by the stock price process $\left(S_{t}\right)$. Our aim is to solve the optimization problem our investor faces, when he tries to find portfolio strategies that maximize the expected utility from terminal wealth. We restrict ourselves to self-financing portfolio strategies and denote by $\pi_{t} \in[0,1]$ the fraction of the wealth invested in the stock at time $t$. The restriction of the fraction to $[0,1]$ (" no short-sellings ") guarantees a positive wealth process which is reasonable for logarithmic and power utility. Due to the jumps of the stock price a violation of this restrictions may lead to a negative wealth with positive probability. The process $\pi=\left(\pi_{t}\right)$ is called portfolio strategy. An admissible portfolio strategy has to be $\mathfrak{F}^{S}$-predictable and takes values in $[0,1]$. Thus, we introduce the set

$$
\mathcal{U}[t, T]:=\left\{\pi=\left(\pi_{s}\right)_{t \leq s \leq T} \mid \pi_{s} \in[0,1] \text { for all } s \in[t, T], \pi \text { is } \mathfrak{F}^{S} \text { - predictable. }\right\} .
$$

The wealth process under an admissible portfolio strategy $\pi \in \mathcal{U}[0, T]$ is given by

$$
d \tilde{X}_{t}^{\pi}=\tilde{X}_{t-}^{\pi}\left(\left(r+(\mu-r) \pi_{t}\right) d t+\sigma \pi_{t} d W_{t}+\pi_{t-} \int y N(d t, d y)\right) .
$$

We assume that $\tilde{X}_{0}^{\pi}=x_{0}$ is the given initial wealth. Let $U: \mathbb{R}_{+} \rightarrow \mathbb{R}$ be an increasing, concave utility function. Then we define the value functions for $\pi \in \mathcal{U}[t, T], t \in[0, T], x>0$ by

$$
\begin{aligned}
\tilde{V}_{\pi}(t, x) & :=E^{t, x}\left[U\left(\tilde{X}_{T}^{\pi}\right) \mid \mathcal{F}_{t}^{S}\right] \\
\tilde{V}(t, x) & :=\sup _{\pi \in \mathcal{U}[t, T]} \tilde{V}_{\pi}(t, x)
\end{aligned}
$$

where the expectation is taken w.r.t. the probability measure $P^{t, x}$ with $\tilde{X}_{t}^{\pi}=x$. Note that $\tilde{V}_{\pi}(t, x)$ and $\tilde{V}(t, x)$ are random variables, in particular $\tilde{V}_{\pi}(t, x)$ is $\mathcal{F}_{t}^{S}$-measurable. Moreover, $\tilde{V}_{\pi}\left(0, x_{0}\right)$ and $\tilde{V}\left(0, x_{0}\right)$ depend on the distribution of $Y_{0}$ which is fixed. A portfolio strategy $\pi^{*} \in \mathcal{U}[0, T]$ is optimal if

$$
\tilde{V}\left(0, x_{0}\right)=\tilde{V}_{\pi^{*}}\left(0, x_{0}\right)
$$


We have chosen the appreciation rate and the volatility to be constant. All the analysis which follows can be done in a similar way if they are modelled by bounded, deterministic (observable) processes.

\section{The Reduction}

We can reduce the control problem to one with complete observation. This procedure is classical. The idea is to update our belief about the distribution of the environment state $Y_{t}$ continuously and make it part of our state space. Note that only the jump time points of the stock price contain relevant information for estimating the environment state and thus the jump intensity. This continuous estimation is done by the so-called Wonham filter. We proceed as in Brémaud (1981) p. 94 ff. Define

$$
p_{k}(t)=P\left(Y_{t}=e_{k} \mid \mathcal{F}_{t}^{S}\right), \quad k=1, \ldots, d
$$

and $p_{t}=\left(p_{1}(t), \ldots, p_{d}(t)\right) \cdot p_{k}(t)$ is the probability that the environment process is in state $k$ at time $t$, given that we have observed the stock price process until time $t$. The process $\left(p_{t}\right)$ is called filter process. Recall form section 2 that the Markov-modulated Poisson process is given by

$$
\int_{0}^{t} \int N(d s, d y)
$$

and counts the number of jumps in the stock price until time $t$. This counting process has $\mathfrak{F}$-intensity $\left(\lambda_{t}\right)=\left(\lambda^{\prime} Y_{t}\right)$ which is equivalent to

$$
\eta_{t}:=\int_{0}^{t} \int N(d s, d y)-\int_{0}^{t} \lambda_{s} d s
$$

being an $\mathfrak{F}$-martingale. The following statements hold:

Lemma 3.1: There exists an $\mathfrak{F}^{S}$-martingale $\left(\hat{\eta}_{t}\right)$ such that

a) the filter processes $p_{k}(t)$ satisfy for $t \geq 0$

$$
d p_{k}(t)=\sum_{j} q_{j k} p_{j}(t) d t+p_{k}(t-)\left(\frac{\lambda_{k}-\hat{\lambda}_{t-}}{\hat{\lambda}_{t-}}\right) d \hat{\eta}_{t}
$$

with $\hat{\lambda}_{t}:=\sum_{k=1}^{d} \lambda_{k} p_{k}(t)=E\left[\lambda_{t} \mid \mathcal{F}_{t}^{S}\right]$.

b) $\lambda_{t} d t+d \eta_{t}=\hat{\lambda}_{t} d t+d \hat{\eta}_{t}$.

c) $\left(W_{t}\right)$ and $\left(\hat{\eta}_{t}\right)$ are independent.

Part b) of Lemma 3.1 states that the Markov-modulated Poisson process $\int_{0}^{t} \int N(d s, d y)$ admits an $\mathfrak{F}^{S}$-intensity $\left(\hat{\lambda}_{t}\right)$ and can be compensated in order to obtain an $\mathfrak{F}^{S}$-martingale

$$
\hat{\eta}_{t}:=\int_{0}^{t} \int N(d s, d y)-\int_{0}^{t} \hat{\lambda}_{s} d s
$$


In what follows we also need the compensated random measure

$$
\hat{M}(d t, d y)=N(d t, d y)-\hat{\lambda}_{t} d t Q(d y) .
$$

Note that

$$
\int_{0}^{t} \int f(s, y) N(d s, d y)-\int_{0}^{t} \int f(s, y) \hat{\lambda}_{s} d s Q(d y)
$$

is a martingale for arbitrary $f$ whenever the integrals exist. The control model with complete observation is now characterized for $\pi \in \mathcal{U}[0, T]$ by the following $d+1$-dimensional state process:

$$
\begin{aligned}
d X_{t}^{\pi} & =X_{t-}^{\pi}\left(\left(r+\pi_{t-}(\mu-r)+\pi_{t-} \hat{\lambda}_{t} \int y Q(d y)\right) d t+\sigma \pi_{t} d W_{t}+\pi_{t-} \int y \hat{M}(d t, d y)\right) \\
X_{0}^{\pi} & =x_{0} \\
d p_{k}(t) & =\sum_{j} q_{j k} p_{j}(t) d t+p_{k}(t-)\left(\frac{\lambda_{k}-\hat{\lambda}_{t-}}{\hat{\lambda}_{t-}}\right) d \hat{\eta}_{t} \\
p_{k}(0) & =P\left(Y_{0}=k\right), \quad k=1, \ldots, d
\end{aligned}
$$

where $P\left(Y_{0}=k\right), \quad k=1, \ldots, d$, is the given distribution of $Y_{0}$. A solution of the stochastic differential equation for the wealth process is given by

$$
\begin{aligned}
X_{t}^{\pi}=x_{0} \exp & \left\{\int_{0}^{t}\left(r+(\mu-r) \pi_{s}-\frac{1}{2} \sigma^{2} \pi_{s}^{2}\right) d s+\int_{0}^{t} \sigma \pi_{s} d W_{s}+\int_{0}^{t} \int \ln \left(1+\pi_{s} y\right) N(d s, d y)\right\} \\
=x_{0} \exp & \left\{\int_{0}^{t}\left(r+(\mu-r) \pi_{s}-\frac{1}{2} \sigma^{2} \pi_{s}^{2}+\hat{\lambda}_{s} \int \ln \left(1+\pi_{s} y\right) Q(d y)\right) d s+\int_{0}^{t} \sigma \pi_{s} d W_{s}\right. \\
+ & \left.\int_{0}^{t} \int \ln \left(1+\pi_{s} y\right) \hat{M}(d s, d y)\right\} .
\end{aligned}
$$

By $\Delta_{d}$ we denote the probability simplex in $\mathbb{R}^{d}$. The value functions in the reduced model are for $\pi \in \mathcal{U}[t, T]$ and $p \in \Delta_{d}, t \in[0, T], x>0$ defined by

$$
\begin{aligned}
V_{\pi}(t, x, p) & :=E^{t, x, p}\left[U\left(X_{T}^{\pi}\right)\right] \\
V(t, x, p) & :=\sup _{\pi \in \mathcal{U}[t, T]} V_{\pi}(t, x, p)
\end{aligned}
$$

where $E^{t, x, p}$ is the conditional expectation, given $X_{t}^{\pi}=x, p_{t}=p$. The reduced model now solves our original problem. This is often taken for granted, however it has to be proved formally. The next theorem states that the filter contains the necessary information in order to solve our original problem. Instead of the whole history $\mathcal{F}_{t}^{S}$ it is sufficient to know $p_{t}$. More precisely, $\tilde{V}(t, x)$ depends on the history $\mathcal{F}_{t}^{S}$ only through $p_{t}$.

Theorem 3.2: For all $\pi \in \mathcal{U}[t, T]$ it holds that $V_{\pi}\left(t, x, p_{t}\right)=\tilde{V}_{\pi}(t, x)$ and $V\left(t, x, p_{t}\right)=\tilde{V}(t, x)$ for all $x>0, t \in[0, T]$.

Proof: From Lemma 3.1 and the stochastic differential equation for the wealth process it follows that $\tilde{X}_{T}^{\pi}=X_{T}^{\pi}$ a.s. for all $\pi \in \mathcal{U}[t, T]$ which obviously yields the statement.

In the reduced model, all processes are $\mathfrak{F}^{S}$-adapted and admit an $\mathfrak{F}^{S}$-intensity respectively. 
Therefore, we can solve this problem by stochastic control techniques. The following properties are easily derived.

\section{Lemma 3.3:}

a) For all $\pi \in \mathcal{U}[t, T], p \in \Delta_{d}$ and $x>0$ we have

$$
V_{\pi}(t, x, p)=\sum_{j=1}^{d} p_{j} V_{\pi}\left(t, x, e_{j}\right)
$$

b) The mapping $p \mapsto V(t, x, p)$ is convex for all $t \in[0, T]$ and $x>0$.

Proof: Part a) is obtained by conditioning.

For b) let $p, q \in \Delta_{d}$ be two initial distributions and $\alpha \in[0,1]$. Then

$$
\begin{aligned}
V(t, x, \alpha p+(1-\alpha) q) & =\sup _{\pi \in \mathcal{U}[t, T]}\left(\alpha \sum_{j} V_{\pi}\left(t, x, e_{j}\right) p_{j}+(1-\alpha) \sum_{j} V_{\pi}\left(t, x, e_{j}\right) q_{j}\right) \\
& \leq \alpha \sup _{\pi \in \mathcal{U}[t, T]} \sum_{j} V_{\pi}\left(t, x, e_{j}\right) p_{j}+(1-\alpha) \sup _{\pi \in \mathcal{U}[t, T]} \sum_{j} V_{\pi}\left(t, x, e_{j}\right) q_{j} \\
& =\alpha V(t, x, p)+(1-\alpha) V(t, x, q) .
\end{aligned}
$$

\section{Logarithmic Utility}

In this section we briefly summarize the results in the case of a logarithmic utility function $U(x)=\log (x)$. This is always the easiest case. For $\pi \in \mathcal{U}[t, T]$ we obtain from the explicit solution for $X_{t}^{\pi}$

$$
V_{\pi}(t, x, p)=\log (x)+h_{\pi}(t, p)
$$

where

$$
h_{\pi}(t, p)=E^{t, p}\left[\int_{t}^{T} r+(\mu-r) \pi_{s}-\frac{1}{2} \sigma^{2} \pi_{s}^{2}+\hat{\lambda}_{s} \int \log \left(1+\pi_{s} y\right) Q(d y) d s\right] .
$$

Note that $h_{\pi}$ does not depend on $x$. Obviously we obtain the following result:

\section{Lemma 4.1:}

a) For all $t \in[0, T], x>0, p \in \Delta_{d}$ we have

$$
V(t, x, p)=\log (x)+h(t, p),
$$

where $h(t, p)=\sup _{\pi \in \mathcal{U}[t, T]} h_{\pi}(t, p)$. 
b) Suppose that for all $p \in \Delta_{d}, u^{*}(p)$ maximizes

$$
u \mapsto r+(\mu-r) u-\frac{1}{2} \sigma^{2} u^{2}+\lambda^{\prime} p \int \log (1+y u) Q(d y) \quad \text { on }[0,1]
$$

then $\pi^{*}=\left(\pi_{t}^{*}\right) \in \mathcal{U}[t, T]$ with $\pi_{t}^{*}=u^{*}\left(p_{t-}\right)$ is an optimal portfolio strategy for the given portfolio problem.

Note that $\pi_{t}^{*}$ depends on $\mathcal{F}_{t}^{S}$ only through $p_{t}$. It is easy to show that in the case of complete observation, i.e. when we know that the state of the Markov chain is for example $e_{i}$, the optimal portfolio strategy would be to invest a constant fraction $u^{*}$ of the wealth in the stock, where $u^{*}$ is the maximizer of

$$
u \mapsto r+(\mu-r) u-\frac{1}{2} \sigma^{2} u^{2}+\lambda_{i} \int \log (1+y u) Q(d y)
$$

on $[0,1]$. Part b) of Lemma 4.1 shows that the so-called certainty equivalence principle holds, i.e. the unknown intensity $\lambda_{t}$ is replaced by the estimate $\hat{\lambda}_{t}=E\left[\lambda_{t} \mid \mathcal{F}_{t}^{S}\right]$ in the optimal portfolio strategy (cf. Kuwana (1991)). This means that uncertainty about the jump intensity does not change the optimal portfolio strategy in this case. The situation is completely different in the case of a power utility function as we will see in section 5.2.

\section{Power Utility}

In this section we assume that the utility function is given by $U(x)=\frac{1}{\gamma} x^{\gamma}$ for $\gamma<1, \gamma \neq 0$. The value function under strategy $\pi \in \mathcal{U}[t, T]$ is therefore

$$
V_{\pi}(t, x, p)=\frac{1}{\gamma} x^{\gamma} g_{\pi}(t, p),
$$

where

$$
\begin{gathered}
g_{\pi}(t, p)=E^{t, p}\left[\operatorname { e x p } \left\{\int_{t}^{T} \gamma\left(r+(\mu-r) \pi_{s}-\frac{1}{2} \sigma^{2} \pi_{s}^{2}\right) d s+\int_{t}^{T} \gamma \sigma \pi_{s} d W_{s}\right.\right. \\
\left.\left.+\gamma \int_{t}^{T} \int \ln \left(1+\pi_{s} y\right) N(d s, d y)\right\}\right] .
\end{gathered}
$$

Note that $g_{\pi}$ does not depend on $x$. If we define

$$
g(t, p):=\sup _{\pi \in \mathcal{U}[t, T]} g_{\pi}(t, p)
$$

then it obviously holds that

$$
V(t, x, p)=\frac{1}{\gamma} x^{\gamma} g(t, p)
$$




\subsection{The Solution of the Portfolio Optimization Problem}

In this section we summarize the main results. We use a stochastic control approach to solve the problem. Unfortunately it is not clear whether the value function is continuously differentiable in $p$ and $t$ and we thus are not able to obtain a classical solution for the associated HamiltonJacobi-Bellman (HJB) equation. The standard way would then be to show that the value function is the unique viscosity solution of the HJB equation. However this type of solution is quite weak and the uniqueness proof can be hard. In our setting it is possible to show that the value function is locally Lipschitz-continuous and thus almost everywhere differentiable. This is much more than continuity which is required for the viscosity solution. Therefore we decided to pursue a different approach by considering a generalized HJB equation where the classical derivative is replaced by a generalized derivative. A similar approach has been used by Davis (1993) for piecewise deterministic models. We can show that the value function is the unique solution of the generalized HJB equation and the maximizer yields an optimal portfolio strategy. In this section we only present the results, proofs are postponed to section 7 .

For the analysis, it is important to note that $\left(p_{t}\right)$ is a piecewise deterministic process with jumps appearing according to the $\mathfrak{F}^{S}$-intensity $\left(\hat{\lambda}_{t}\right)$. We denote by

$$
\phi_{k}\left(t, p_{0}\right)=p_{k}(0)+\int_{0}^{t} \sum_{j} q_{j k} p_{j}(s)-p_{k}(s)\left(\lambda_{k}-\hat{\lambda}_{s}\right) d s, \quad k=1, \ldots, d
$$

and $\phi\left(t, p_{0}\right)=\left(\phi_{1}\left(t, p_{0}\right), \ldots, \phi_{d}\left(t, p_{0}\right)\right)$ the evolution of the filter between jumps and by

$$
J(p)=\left(\frac{\lambda_{1} p_{1}}{\lambda^{\prime} p}, \ldots, \frac{\lambda_{d} p_{d}}{\lambda^{\prime} p}\right)
$$

the new state of the filter directly after a jump from state $p$. Moreover, we let $\mathcal{S}_{d}$ be the interior of the probability simplex $\Delta_{d}$. In order to obtain a reasonable model we assume now that all states of the Markov chain $Y$ communicate. Thus, the filter process $p_{t}$ will for $t>0$ always stay in $\mathcal{S}_{d}$. Let us introduce the following operator $L$ which acts on functions $v:[0, T] \times \mathcal{S}_{d} \rightarrow \mathbb{R}$ and $u \in[0,1]$

$$
\begin{aligned}
L v(t, p, u):= & v(t, p)\left(r+(\mu-r) u+\frac{1}{2}(\gamma-1) \sigma^{2} u^{2}\right) \\
& +\frac{\lambda^{\prime} p}{\gamma}\left(v(t, J(p)) \int(1+y u)^{\gamma} Q(d y)-v(t, p)\right) .
\end{aligned}
$$

In order to motivate the HJB equation of this problem, we give some heuristic arguments. For this purpose, suppose that the value function $V$ is sufficiently differentiable. An application of Ito's Lemma gives:

$$
\begin{aligned}
V\left(T, X_{T}^{\pi}, p_{T}\right)= & V(t, x, p)+\int_{t}^{T} V_{t}\left(s, X_{s}^{\pi}, p_{s}\right) d s+\int_{t}^{T} V_{x}\left(s, X_{s}^{\pi}, p_{s}\right) d X_{s}^{\pi} \\
& +\sum_{k=1}^{d} \int_{t}^{T} V_{p_{k}}\left(s, X_{s}^{\pi}, p_{s}\right) d p_{k}(s)+\frac{1}{2} \int_{t}^{T} V_{x x}\left(s, X_{s}^{\pi}, p_{s}\right) \sigma^{2}\left(X_{s}^{\pi}\right)^{2} \pi_{s}^{2} d s \\
& +\sum_{0<s \leq t}\left[V\left(s, X_{s}^{\pi}, p_{s}\right)-V\left(s-, X_{s-}^{\pi}, p_{s-}\right)\right]-V_{x}\left(s, X_{s}^{\pi}, p_{s}\right) \Delta X_{s}^{\pi} \\
& -\sum_{k=1}^{d} V_{p_{k}}\left(s, X_{s}^{\pi}, p_{s}\right) \Delta p_{k}(s) .
\end{aligned}
$$


It can be shown that $t \mapsto V\left(t, X_{t}^{\pi}, p_{t}\right)$ is a martingale under the optimal portfolio strategy and a supermartingale under any admissible strategy. Thus, the drift terms in the preceding equation have to be zero. Moreover, plugging in the form $V(t, x, p)=\frac{1}{\gamma} x^{\gamma} g(t, p)$ yields as an optimality condition:

$$
\begin{aligned}
0= & \frac{1}{\gamma} g_{t}(t, p)+g(t, p)\left(r+\pi(\mu-r)+\frac{1}{2}(\gamma-1) \sigma^{2} \pi^{2}\right) \\
& +\frac{\lambda^{\prime} p}{\gamma}\left(g(t, J(p)) \int(1+y \pi)^{\gamma} Q(d y)-g(t, p)\right) \\
& +\frac{1}{\gamma} \sum_{k=1}^{d} g_{p_{k}}(t, p)\left(\sum_{j} q_{j k} p_{j}-p_{k}\left(\lambda_{k}-\lambda^{\prime} p\right)\right) .
\end{aligned}
$$

However, since the value function (in particular $g$ defined in equation (1)) is probably not differentiable w.r.t. $g_{k}$ we replace the gradient by the Clarke generalized gradient. The resulting generalized Hamilton-Jacobi-Bellman equation for our problem then reads as follows

$$
0=\sup _{u \in[0,1]}\{L g(t, p, u)\}+\sup _{\theta \in \partial g(t, p)}\left\{\frac{1}{\gamma} \theta_{0}+\frac{1}{\gamma} \sum_{k=1}^{d} \theta_{k}\left(\sum_{j} q_{j k} p_{j}-p_{k}\left(\lambda_{k}-\lambda^{\prime} p\right)\right)\right\}
$$

with boundary condition $g(T, p)=1$ for all $p \in \mathcal{S}_{d}$. The set $\partial g(t, p) \subset \mathbb{R}^{d+1}$ denotes the Clarke generalized gradient (see Clarke (1983)). This is a weaker notion for differentiability which is defined as follows: let $f: \mathbb{R}^{d} \rightarrow \mathbb{R}$ be a locally Lipschitz continuous function. For $x, y \in \mathbb{R}^{d}$ the upper generalized directional derivative of $f$ at $x$ in direction $y$ is defined by

$$
f^{0}(x ; y):=\limsup _{z \rightarrow x, \varepsilon \rightarrow 0} \frac{f(z+\varepsilon y)-f(z)}{\varepsilon} .
$$

The Clarke generalized gradient of $f$ at $x$ is now defined by the set

$$
\partial f(x):=\left\{\theta \in \mathbb{R}^{n} \mid f^{0}(x ; y) \geq \theta y \text { for all } y \in \mathbb{R}^{d}\right\} .
$$

$\partial f(x)$ is a non-empty, convex, compact subset of $\mathbb{R}^{d}$ and if $f$ is differentiable at $x$, then $\partial f(x):=\{\nabla f(x)\}$. Moreover, since $f$ is locally Lipschitz continuous, it is almost everywhere differentiable and we can find for every point $x \in \mathbb{R}^{d}$ sequences of points $x_{n} \in \mathbb{R}^{d}$ such that $\lim _{n \rightarrow \infty} x_{n}=x$ and $f$ is differentiable at $x_{n}$. $\partial f(x)$ can then be written as the closed convex hull of existing limits of sequences $\nabla f\left(x_{n}\right)$, i.e.

$$
\partial f(x):=c o\left\{\limsup _{n \rightarrow \infty} \nabla f\left(x_{n}\right) \mid \lim _{n \rightarrow \infty} x_{n}=x\right\} .
$$

Our first result is a verification theorem:

Theorem 5.1: Suppose there exists a bounded function $v:[0, T] \times \mathcal{S}_{d} \rightarrow \mathbb{R}_{+}$such that for all $p \in \mathcal{S}_{d}, t \mapsto v(t, \phi(t, p))$ is absolutely continuous, $v(T, p)=1$ and $v$ satisfies the generalized HJB equation. Further assume that $u^{*}$ is a maximizer of the generalized HJB equation, i.e. for all $t \in[0, T]$ and $p \in \mathcal{S}_{d}, u^{*}(t, p)$ maximizes

$$
u \mapsto L v(t, p, u) \quad \text { on }[0,1] .
$$


Then $V(t, x, p)=\frac{1}{\gamma} x^{\gamma} v(t, p)$ and the strategy $\pi^{*}=\left(\pi_{t}^{*}\right) \in \mathcal{U}[0, T]$ with $\pi_{t}^{*}:=u^{*}\left(t-, p_{t-}\right)$ is an optimal feedback strategy for the given portfolio problem.

Note that $\pi_{t}^{*}$ depends on $\mathcal{F}_{t}^{S}$ only through $p_{t}$. The next theorem states the existence of a solution of the generalized HJB equation.

Theorem 5.2: The value function of our problem is given by $V(t, x, p)=\frac{1}{\gamma} x^{\gamma} g(t, p)$ with $g$ defined by (1) above and $g$ satisfies the generalized HJB equation

$$
0=\sup _{u \in[0,1]}\{L g(t, p, u)\}+\sup _{\theta \in \partial g(t, p)}\left\{\frac{1}{\gamma} \theta_{0}+\frac{1}{\gamma} \sum_{k=1}^{d} \theta_{k}\left(\sum_{j} q_{j k} p_{j}-p_{k}\left(\lambda_{k}-\lambda^{\prime} p\right)\right)\right\}
$$

with boundary condition $g(T, p)=1$ for all $p \in \mathcal{S}_{d}$. Moreover, $\pi^{*}$ from Theorem 5.1 (with $v$ replaced by $g$ ) is an optimal portfolio strategy.

\subsection{Special Cases and Properties of the Optimal Portfolio Strategy}

In this section we investigate the optimal portfolio strategy in some special cases in greater detail and establish some interesting properties. In particular we discuss the influence of uncertainty on the optimal portfolio strategy.

\section{A) Jumps occur with known and constant intensity}

Suppose that $\delta_{n} \equiv \delta \in(-1, \infty)$ is deterministic and that the jumps in the stock price process occur with known constant intensity $\lambda>0$, i.e. $\lambda=\lambda_{1}=\ldots=\lambda_{d}$. This model is similar to the setup investigated in Øksendal and Sulem (2004) and Framstad et al. (1999). In this case it is optimal to invest a constant fraction $u_{\delta}^{*}(\lambda)$ (independent of time) of the wealth in the stock. Specializing our HJB equation (note that $J(p)=p$ in this case), it is easy to see that $u_{\delta}^{*}(\lambda)$ is the maximum point of the mapping

$$
u \mapsto(\mu-r) u+\frac{1}{2}(\gamma-1) \sigma^{2} u^{2}+\frac{\lambda}{\gamma}(1+\delta u)^{\gamma} \quad \text { on }[0,1] .
$$

In this case it can also be shown that the value function is a classical solution of the HJB equation.

In what follows we want to compare the optimal fractions which are invested in the stock in different models. In particular we highlight the role of uncertainty. For this task the following simple lemma is useful:

Lemma 5.3: Let $f, h:[0,1] \rightarrow \mathbb{R}$ be continuous functions and suppose that $h$ is increasing. If we denote

$$
\begin{aligned}
u_{f}^{*} & :=\operatorname{argmax}\{f(u) \mid u \in[0,1]\} \\
u_{f+h}^{*} & :=\operatorname{argmax}\{f(u)+h(u) \mid u \in[0,1]\}
\end{aligned}
$$


then $u_{f}^{*} \leq u_{f+h}^{*}$.

Throughout the paper we use increasing and decreasing in the non-strict sense. A direct implication of the previous lemma is

Lemma 5.4: If $\delta<0$, then $\lambda \mapsto u_{\delta}^{*}(\lambda)$ is decreasing and if $\delta>0$, then $\lambda \mapsto u_{\delta}^{*}(\lambda)$ is increasing.

Of course this result is not surprising. If we have downward jumps, we invest less in the stock if the jump intensity increases. In order to investigate the influence of further uncertainty we have to add a jump martingale to the stock price to keep the expected drift unchanged. Thus, suppose for a moment that the stock price process evolves according to

$$
d S_{t}=S_{t-}\left(\hat{\mu} d t+\sigma d W_{t}+\delta d \eta_{t}\right),
$$

where $\eta_{t}=\int_{0}^{t} \int N(d s, d y)-\lambda t$. If we set $\hat{\mu}=\mu+\lambda \delta$ we obtain a stochastic differential equation for the stock price in the form given in section 1 . In the case without jumps $(\delta=0)$, we know that the optimal fraction maximizes

$$
u \mapsto(\hat{\mu}-r) u+\frac{1}{2}(\gamma-1) \sigma^{2} u^{2} \quad \text { on }[0,1] .
$$

In the case with jumps $(\delta \neq 0)$, we know that the optimal fraction maximizes

$$
u \mapsto(\hat{\mu}-r) u+\frac{1}{2}(\gamma-1) \sigma^{2} u^{2}+\frac{\lambda}{\gamma}(1+\delta u)^{\gamma}-\lambda \delta u \quad \text { on }[0,1] .
$$

Thus we obtain the following comparison result:

Theorem 5.5: In the previous model we have

$$
u_{0}^{*} \geq u_{\delta}^{*}(\lambda)
$$

Proof: In view of Lemma 5.3 it is sufficient to show that

$$
h(u):=\frac{\lambda}{\gamma}(1+\delta u)^{\gamma}-\lambda \delta u
$$

is decreasing for all $\delta>-1, \delta \neq 0$. This can be done by showing that $h^{\prime}(u) \leq 0$.

Theorem 5.5 means that the optimal fraction invested in the stock in the model with further uncertainty coming from jumps is always less or equal to the optimal fraction in the model without jumps. Note that the expected drift of the stock remains the same in both scenarios. Since we have a risk averse investor such a result is not unexpected. However also note that the statement is true for all $\gamma<1, \gamma \neq 0$. In B) we will observe a different behavior.

\section{B) Jumps occur with unknown and constant intensity - the Bayesian case}

Suppose that $\delta_{n} \equiv \delta \in(-1, \infty)$ is deterministic and that the jumps in the stock price process occur with unknown constant intensity $\lambda>0$. We assume that $\lambda$ can be one of the possible 
values $\lambda_{1} \leq \ldots \leq \lambda_{d}$ and that the initial probability $p_{0} \in \mathcal{S}_{d}$ for the values is given. Thus, we have a Bayesian control problem with an unknown parameter. This is a special case of our model, if we formally set the intensity matrix of the Markov chain $\left(Y_{t}\right)$ to zero, i.e. $Q_{0}=0$ and the Markov chain stays in the initial state. If we define

$$
p_{k}(t)=P\left(Y_{0}=e_{k} \mid \mathfrak{F}_{t}^{S}\right)=P\left(\lambda=\lambda_{k} \mid \mathfrak{F}_{t}^{S}\right)
$$

and $p_{t}=\left(p_{1}(t), \ldots, p_{d}(t)\right)$, then the following equation holds

$$
p_{k}(t)=p_{k}(0)+\int_{0}^{t} p_{k}(s-)\left(\frac{\lambda_{k}-\hat{\lambda}_{s-}}{\hat{\lambda}_{s-}}\right) d \hat{\eta}_{s}
$$

where $\left(\hat{\eta}_{t}\right)$ is defined as in Lemma 3.1. The optimal fraction $\pi_{t}^{*}$ invested in the stock depends on the time and the estimate $p_{t-}$, i.e $\pi_{t}^{*}=u_{\delta}^{*}\left(t-, p_{t-}\right)$ and $u_{\delta}^{*}$ maximizes

$$
u \mapsto(\mu-r) u+\frac{1}{2}(\gamma-1) \sigma^{2} u^{2}+\frac{\lambda^{\prime} p}{\gamma} \frac{g(t, J(p))}{g(t, p)}(1+\delta u)^{\gamma} \quad \text { on }[0,1] .
$$

It is possible to compare the optimal portfolio strategy of this scenario with the previous case A) of complete observation.

Theorem 5.6: The optimal fraction $u_{\delta}^{*}(t, p)$ invested in the stock has the following properties:

a) If $\delta<0$ (downward jumps) it holds for all $(t, p) \in[0, T] \times \mathcal{S}_{d}$ that

$$
u_{\delta}^{*}\left(\lambda_{d}\right) \leq u_{\delta}^{*}(t, p) \leq u_{\delta}^{*}\left(\lambda_{1}\right) .
$$

If $\delta>0$ (upward jumps) the inequalities are reversed.

b) If $\delta \gamma<0$ it holds for all $(t, p) \in[0, T] \times \mathcal{S}_{d}$ that

$$
u_{\delta}^{*}\left(\lambda^{\prime} p\right) \leq u_{\delta}^{*}(t, p)
$$

If $\delta \gamma>0$ the inequality is reversed.

Proof:

a) Suppose $\delta<0$. In view of Lemma 5.3 it suffices to show

$$
\lambda^{\prime} p \cdot g(t, J(p)) \geq \lambda_{1} g(t, p) \quad \text { and } \quad \lambda^{\prime} p \cdot g(t, J(p)) \leq \lambda_{d} g(t, p) .
$$

Recall that

$$
g(t, p)=\sup _{\pi \in \mathcal{U}[t, T]} g_{\pi}(t, p)=\sup _{\pi \in \mathcal{U}[t, T]} \sum_{j=1}^{d} p_{j} g_{\pi}\left(t, e_{j}\right) .
$$

Now suppose $\pi \in \mathcal{U}[t, T]$ is fix. Note that due to the definition $g_{\pi}\left(t, e_{j}\right) \geq 0$ for all $t \in[0, T]$ and $j$. We obtain

$$
\lambda^{\prime} p \cdot g_{\pi}(t, J(p))=\sum_{j=1}^{d} p_{j} \lambda_{j} g_{\pi}\left(t, e_{j}\right) \geq \lambda_{1} \sum_{j=1}^{d} p_{j} g_{\pi}\left(t, e_{j}\right)=\lambda_{1} g_{\pi}(t, p) .
$$

Taking the supremum over all $\pi \in \mathcal{U}[t, T]$ then yields the first inequality. The case $\delta>0$ and the second statement obviously follows similarly. 
b) Suppose $\delta<0$ and $0<\gamma<1$. In view of Lemma 5.3 it suffices to show

$$
g(t, J(p)) \leq g(t, p) .
$$

Note that if $p=e_{j}$, the counting process of jumps is simply a Poisson process with intensity $\lambda_{j}$. Moreover, it is well-known that if $\lambda \geq \hat{\lambda}>0$, then a Poisson process with intensity $\lambda$ pathwise stochastically dominates a Poisson process with intensity $\hat{\lambda}$ (see e.g. Sec. 4.3.3 in Müller and Stoyan (2002)). Thus, under an arbitrary fixed $\pi \in \mathcal{U}[t, T]$ we have $X_{t}^{\pi} \leq_{s t} \hat{X}_{t}^{\pi}$ where $\leq_{s t}$ is the usual stochastic order. Thus, the value function is decreasing in $\lambda$ and we obtain

$$
g_{\pi}\left(t, e_{1}\right) \geq \ldots \geq g_{\pi}\left(t, e_{d}\right),
$$

for all $t \in[0, T]$. Thus, it follows that

$$
\sum_{j=1}^{d} p_{j} g_{\pi}\left(t, e_{j}\right) \sum_{j=1}^{d} p_{j} \lambda_{j} \geq \sum_{j=1}^{d} p_{j} \lambda_{j} g_{\pi}\left(t, e_{j}\right)
$$

where this inequality is derived by applying the following general inequality (cf. Mitronovic et al. (1993)): let $\alpha_{1} \leq \ldots \leq \alpha_{d}$ and $\beta_{1} \geq \ldots \geq \beta_{d}$ be real numbers and $p_{1}, \ldots, p_{d} \geq$ $0, \sum_{j=1}^{d} p_{j}=1$. Then

$$
\sum_{j=1}^{d} p_{j} \alpha_{j} \sum_{j=1}^{d} p_{j} \beta_{j} \geq \sum_{j=1}^{d} p_{j} \alpha_{j} \beta_{j} .
$$

Taking the supremum over all $\pi \in \mathcal{U}[t, T]$ then yields the statement.

Please note that in the case $\gamma<0$ we obtain the inequality

$$
g_{\pi}\left(t, e_{1}\right) \leq \ldots \leq g_{\pi}\left(t, e_{d}\right),
$$

for all $t \in[0, T]$ since the value function is negative. The case $\delta>0$ can be shown analogously.

Part a) of Theorem 5.6 means that the optimal fraction which is invested in the stock is bounded by the smallest and largest invested fraction in the models with known intensity $\lambda_{1}$ and $\lambda_{d}$. Part b) of this theorem is most interesting. For example in the case of downward jumps $\delta<0$ and $\gamma \in(0,1)$, the optimal fraction invested in the stock in the model with unknown jump intensity in state $(t, p)$ is larger than in the model with known (average) intensity $\lambda^{\prime} p$. Though our investor is risk averse, this is a situation where more uncertainty leads to a higher investment in the risky stock. If $\gamma<0$ the situation is vice versa. An economic explanation is that the degree of risk aversion changes with $\gamma$. From the Arrow-Pratt absolute risk aversion coefficient which is

$$
-\frac{U^{\prime \prime}(x)}{U^{\prime}(x)}=(1-\gamma) \frac{1}{x}
$$

in the case of the power utility $U(x)=\frac{1}{\gamma} x^{\gamma}$, we see that the risk aversion decreases with $\gamma$ for all wealth levels. If $\gamma \rightarrow 0$ we obtain the logarithmic utility case and we know from section 4 that here the optimal fractions invested coincide, i.e. $u_{\delta}^{*}\left(\lambda^{\prime} p\right)=u_{\delta}^{*}(t, p)$. In particular if $\gamma \in(0,1)$ the investor is less risk averse. A similar result has been obtained for a model with unobservable appreciation rate in Rieder and Bäuerle (2005). 


\section{Auxiliary Results}

In this section we summarize some results which are important for the proofs of our main theorems. Lemma 6.1 summarizes important properties of the function $g$ defined in (1) which is part of the value function $V$.

Lemma 6.1: Let $g$ be defined by (1) in Section 5.

a) $p \mapsto g(t, p)$ is convex for all $t \in[0, T]$.

b) $t \mapsto g(t, p)$ is decreasing (increasing) for all $p \in \mathcal{S}_{d}$ if $0<\gamma<1,(\gamma<0)$.

c) $g(t, p)$ is bounded on $[0, T] \times \mathcal{S}_{d}$.

d) $t \mapsto g(t, p)$ is locally Lipschitz-continuous for all $p \in \mathcal{S}_{d}$.

e) $t \mapsto g(t, \phi(t, p))$ is locally Lipschitz-continuous for all $p \in \mathcal{S}_{d}$.

Proof:

a) follows from Lemma $3.3 \mathrm{~b})$.

b) This is equivalent to showing that $t \mapsto V(t, x, p)$ is decreasing. But this is clear since because of $r>0$ we get a positive reward over a small time interval by putting all the money in the stock.

c) For $\gamma<0$ the statement is obvious due to part b) and the fact that $g(t, p) \geq 0$ and $g(T, p)=1$. For $\gamma \in(0,1)$ it suffices to show that $g(0, p)$ is bounded on $\mathcal{S}_{d}$. It is convenient to introduce a new measure $Q^{\pi}$ by $d Q^{\pi}=L_{T}^{\pi} d P$, where $\pi \in \mathcal{U}[0, T]$ and $L_{t}^{\pi}$ is a solution of the stochastic differential equation

$$
d L_{t}^{\pi}=L_{t-}^{\pi}\left(\gamma \sigma \pi_{t} d W_{t}+\int\left(\left(1+y \pi_{t}\right)^{\gamma}-1\right) \hat{M}(d t, d y)\right)
$$

where $\hat{M}(d t, d y):=N(d t, d y)-\hat{\lambda}_{t} d t Q(d y)$ is the compensated random measure defined before. The solution is given by

$$
\begin{aligned}
L_{T}^{\pi}=\exp \left\{\int_{0}^{T}(\right. & \left.-\frac{1}{2} \gamma^{2} \sigma^{2} \pi_{s}^{2}-\hat{\lambda}_{s} \int\left(\left(1+y \pi_{s}\right)^{\gamma}-1\right) Q(d y)\right) d s+\int_{0}^{T} \gamma \sigma \pi_{s} d W_{s} \\
& \left.+\gamma \int_{0}^{T} \int \ln \left(1+y \pi_{s}\right) N(d s, d y)\right\} .
\end{aligned}
$$

It is easy to see that for $\pi \in \mathcal{U}[0, T]$

$$
\begin{aligned}
g_{\pi}(0, p)=E_{Q^{\pi}}^{0, p}[\exp \{ & \int_{0}^{T} \gamma\left(r+(\mu-r) \pi_{s}+\frac{1}{2}(\gamma-1) \sigma^{2} \pi_{s}^{2}\right) \\
& \left.\left.+\hat{\lambda}_{s} \int\left(\left(1+y \pi_{s}\right)^{\gamma}-1\right) Q(d y) d s\right\}\right] .
\end{aligned}
$$

Since $\pi_{t}, \delta_{n}$ and $\hat{\lambda}_{t}=\lambda^{\prime} p_{t}$ are bounded, it follows from this equation that $g_{\pi}(t, p)$ is bounded on $[0, T] \times \mathcal{S}_{d}$ and the bound is independent of $\pi$. 
d) In this part we make the dependence of $g$ on the time horizon explicit by writing $g_{\pi, T}(t, p)$. First note the following: if $\pi \in \mathcal{U}[t, T]$ we define $\hat{\pi}$ by $\hat{\pi}_{s}=\pi_{t+s}$ for $s \in[0, T-t]$ which implies that $g_{\pi, T}(t, p)=g_{\hat{\pi}, T-t}(0, p)$. Now let $0 \leq t_{1}<t_{2} \leq T$. Then there exists for every $\varepsilon>0$ a strategy $\pi \in \mathcal{U}\left[0, T-t_{1}\right]$ with

$$
\begin{aligned}
& \left|g\left(t_{1}, p\right)-g\left(t_{2}, p\right)\right| \leq\left|g_{\pi, T-t_{1}}(0, p)-g_{\pi, T-t_{2}}(0, p)\right|+\varepsilon \\
\leq & K_{0} E_{Q^{\pi}}^{0, p}\left[\mid \exp \left\{\int_{T-t_{2}}^{T-t_{1}} \gamma\left(r+(\mu-r) \pi_{s}+\frac{1}{2}(\gamma-1) \sigma^{2} \pi_{s}^{2}\right)\right.\right. \\
& \left.\left.+\hat{\lambda}_{s} \int\left(\left(1+y \pi_{s}\right)^{\gamma}-1\right) Q(d y) d s\right\}-1 \mid\right]+\varepsilon \\
\leq & K_{1} E_{Q^{\pi}}^{0, p}\left[\int_{T-t_{2}}^{T-t_{1}}|\gamma| \cdot\left|2 r+\mu+\sigma^{2}\right|+\bar{\lambda} \int\left(\left(1+y \pi_{s}\right)^{\gamma}+1\right) Q(d y) d s\right]+\varepsilon \\
\leq & K_{2}\left|t_{2}-t_{1}\right|+\varepsilon
\end{aligned}
$$

where $\bar{\lambda}=\max _{k} \lambda_{k}$. This implies the statement if we let $\varepsilon \rightarrow 0$. Note that $K_{2}$ can be chosen independent of $p$ and $\pi$.

e) Let $0 \leq t_{1}<t_{2} \leq T$. Then

$$
\begin{aligned}
\left|g\left(t_{2}, \phi\left(t_{2}, p\right)\right)-g\left(t_{1}, \phi\left(t_{1}, p\right)\right)\right|= & \mid g\left(t_{2}, \phi\left(t_{2}, p\right)\right)-g\left(t_{2}, \phi\left(t_{1}, p\right)\right)+ \\
& g\left(t_{2}, \phi\left(t_{1}, p\right)\right)-g\left(t_{1}, \phi\left(t_{1}, p\right)\right) \mid \\
\leq & \left|g\left(t_{2}, \phi\left(t_{2}, p\right)\right)-g\left(t_{2}, \phi\left(t_{1}, p\right)\right)\right| \\
+ & \left|g\left(t_{2}, \phi\left(t_{1}, p\right)\right)-g\left(t_{1}, \phi\left(t_{1}, p\right)\right)\right| .
\end{aligned}
$$

Since $g$ is convex in $p$ it is also locally Lipschitz-continuous in $p \in \mathcal{S}_{d}$ with a module $K_{3}$ which can be chosen independent of $t$ (see e.g. Sec. 10 in Rockafellar (1970)). Therefore we obtain

$$
\left|g\left(t_{2}, \phi\left(t_{2}, p\right)\right)-g\left(t_{1}, \phi\left(t_{1}, p\right)\right)\right| \leq K_{3}\left\|\phi\left(t_{2}, p\right)-\phi\left(t_{1}, p\right)\right\|+K_{2}\left|t_{2}-t_{1}\right| .
$$

But obviously $t \mapsto \phi(t, p)$ is also locally Lipschitz-continuous with a module independent of $p$ which yields the result.

Recall that $0=T_{0}<T_{1}<T_{2}<\ldots$ are the jump time points of the Markov-modulated Poisson process. Since $t \mapsto g(t, \phi(t, p))$ is locally Lipschitz-continuous, there exists a function $D g\left(s, p_{s}\right)$ such that

$$
g\left(T_{i-}, p_{T_{i-}}\right)-g\left(T_{i-1}, p_{T_{i-1}}\right)=\int_{T_{i-1}}^{T_{i}} D g\left(s, p_{s}\right) d s
$$

Almost everywhere on the time interval $[0, T]$, the derivative of $g\left(s, p_{s}\right)$ w.r.t. $s$ exists and we can choose $D g\left(s, p_{s}\right)=g_{t}\left(s, p_{s}\right)+\sum_{k} g_{p_{k}}\left(s, p_{s}\right) \dot{\phi}_{k}(s, p)$. Let us define the operator

$$
H v(t, p, u):=L v(t, p, u)+\frac{1}{\gamma} D v(t, p)
$$


for all functions $v:[0, T] \times \mathcal{S}_{d} \rightarrow \mathbb{R}$ where the right-hand side is well defined. Note that the HJB equation can be written as

$$
0=\sup _{u \in[0,1]}\{H v(t, p, u)\}=\sup _{u \in[0,1]}\{L v(t, p, u)\}+\frac{1}{\gamma} D v(t, p)
$$

at those points $(t, p)$ where $v$ is differentiable.

Lemma 6.2: Suppose that $\pi \in \mathcal{U}[0, T]$ is an arbitrary strategy. The value function $V$ satisfies the following stochastic differential equation

$$
d V\left(t, X_{t}^{\pi}, p_{t}\right)=\left(X_{t}^{\pi}\right)^{\gamma} \cdot H g\left(t, p_{t}, \pi_{t}\right) d t+d \eta_{t}^{\pi},
$$

where $\left(\eta_{t}^{\pi}\right)$ is an $\mathfrak{F}^{S}$-martingale with zero expectation.

Proof: Let $\pi \in \mathcal{U}[0, T]$ be arbitrary. Using Ito's Lemma we can verify that $Z_{t}^{\pi}:=\left(X_{t}^{\pi}\right)^{\gamma}$ satisfies the following stochastic differential equation

$$
\begin{aligned}
d Z_{t}^{\pi}= & \gamma Z_{t}^{\pi}\left(r+(\mu-r) \pi_{t}+\frac{1}{2}(\gamma-1) \sigma^{2} \pi_{t}^{2}\right) d t \\
& +\gamma Z_{t}^{\pi} \sigma \pi_{t} d W_{t}+Z_{t-}^{\pi} \int\left(\left(1+y \pi_{t-}\right)^{\gamma}-1\right) N(d t, d y) .
\end{aligned}
$$

Moreover, since $t \mapsto g(t, \phi(t, p))$ is absolutely continuous, we can write

$$
g\left(t, p_{t}\right)=g\left(0, p_{0}\right)+\int_{0}^{t} D g\left(s, p_{s}\right) d s+\sum_{0<s \leq t} g\left(s, p_{s}\right)-g\left(s-, p_{s-}\right) .
$$

Since $V\left(t, X_{t}^{\pi}, p_{t}\right)=\frac{1}{\gamma} Z_{t}^{\pi} \cdot g\left(t, p_{t}\right)$, the product rule implies

$$
\begin{aligned}
V\left(t, X_{t}^{\pi}, p_{t}\right)= & V(0, x, p)+\int_{0}^{t} g\left(s, p_{s}\right) Z_{s}^{\pi}\left(r+(\mu-r) \pi_{s}+\frac{1}{2}(\gamma-1) \sigma^{2} \pi_{s}^{2}\right) d s \\
& +\int_{0}^{t} g\left(s, p_{s}\right) Z_{s}^{\pi} \sigma \pi_{s} d W_{s}+\int_{0}^{t} Z_{s}^{\pi} \frac{1}{\gamma} D g\left(s, p_{s}\right) d s \\
& +\frac{1}{\gamma} \sum_{0<s \leq t} Z_{s}^{\pi} g\left(s, p_{s}\right)-Z_{s-}^{\pi} g\left(s-, p_{s-}\right) .
\end{aligned}
$$

Let us define

$$
\begin{aligned}
\eta_{t}^{\pi, 1}:= & \frac{1}{\gamma} \sum_{0<s \leq t} Z_{s}^{\pi} g\left(s, p_{s}\right)-Z_{s-}^{\pi} g\left(s-, p_{s-}\right) \\
& -\frac{1}{\gamma} \int_{0}^{t} Z_{s}^{\pi} \hat{\lambda}_{s}\left(g\left(s, J\left(p_{s}\right)\right) \int\left(1+y \pi_{s}\right)^{\gamma} Q(d y)-g\left(s, p_{s}\right)\right) d s .
\end{aligned}
$$

According to Brémaud p.27, T8, $\left(\eta_{t}^{\pi, 1}\right)$ is an $\mathfrak{F}^{S}$-martingale, since

$$
E\left[\int_{0}^{T}\left|Z_{s}^{\pi} g\left(s, p_{s}\right)-Z_{s-}^{\pi} g\left(s-, p_{s-}\right)\right| \lambda^{\prime} p_{s} d s\right]<\infty .
$$


Moreover, due to our boundedness conditions

$$
\eta_{t}^{\pi, 2}:=\int_{0}^{t} g\left(s, p_{s}\right) Z_{s}^{\pi} \sigma \pi_{s} d W_{s}
$$

is also an $\mathfrak{F}^{S}$-martingale. If we define now $\eta_{t}^{\pi}:=\eta_{t}^{\pi, 1}+\eta_{t}^{\pi, 2}$ the statement follows.

Theorem 6.3: Bellman equation

Let $\tau \in[t, T]$ be an $\mathfrak{F}^{S}$-stopping time. Then

$$
V(t, x, p)=\sup _{\pi \in \mathcal{U}[t, T]} E^{t, x, p}\left[V\left(\tau, X_{\tau}^{\pi}, p_{\tau}\right)\right]
$$

The proof of the Bellman equation follows the usual recipe and we skip it here.

\section{Proofs for the Results in Section 5.1}

In this section we provide the proofs of the Verification Theorem and the fact that $V$ is a solution of the generalized HJB equation.

Proof of Theorem 5.1:

Let $\pi \in \mathcal{U}[0, T]$ be an arbitrary strategy. Then we obtain for $Z_{t}^{\pi}:=\left(X_{t}^{\pi}\right)^{\gamma}$ and $G(t, x, p):=$ $\frac{1}{\gamma} x^{\gamma} v(t, p)$ as in Lemma 6.2

$$
G\left(T, X_{T}^{\pi}, p_{T}\right)=G(t, x, p)+\int_{t}^{T} Z_{s}^{\pi} \cdot H v\left(s, p_{s}, \pi_{s}\right) d s+\eta_{T}^{\pi}-\eta_{t}^{\pi}
$$

where $\left(\eta_{t}^{\pi}\right)$ is an $\mathfrak{F}^{S}$-martingale with zero expectation. At those points where $v\left(s, p_{s}\right)$ is differentiable we have $H v\left(s, p_{s}, \pi_{s}\right) \leq 0$ since $v$ satisfies the generalized HJB equation. Moreover, $s \mapsto v\left(s, p_{s}\right)$ is almost everywhere differentiable which yields

$$
\int_{0}^{T} Z_{s}^{\pi} \cdot H v\left(s, p_{s}, \pi_{s}\right) d s \leq 0
$$

Thus, we obtain

$$
G\left(T, X_{T}^{\pi}, p_{T}\right) \leq G(t, x, p)+\eta_{T}^{\pi}-\eta_{t}^{\pi} .
$$

Taking the conditional expectation on both sides (note that $G\left(T, X_{T}^{\pi}, p_{T}\right)=U\left(X_{T}^{\pi}\right)$ ) yields:

$$
E^{t, x, p}\left[U\left(X_{T}^{\pi}\right)\right] \leq G(t, x, p) .
$$

Taking the supremum over all admissible strategies gives $V(t, x, p) \leq G(t, x, p)$. Next, note that the maximum points of the HJB equation trivially exist. If we use $\pi^{*}$ we obtain

$$
\int_{t}^{T} Z_{s}^{\pi^{*}} \cdot H v\left(s, p_{s}, \pi_{s}^{*}\right) d s=0
$$


and the result follows.

Proof of Theorem 5.2:

Let $\tau$ be the time of the first jump of the stock price process $\left(S_{t}\right)$ after time $t$. From the Bellman equation (Theorem 6.3) we obtain for every strategy $\pi \in \mathcal{U}[t, T]$ and $t<t^{\prime} \leq T$ :

$$
V(t, x, p) \geq E^{t, x, p}\left[V\left(\tau \wedge t^{\prime}, X_{\tau \wedge t^{\prime}}^{\pi}, p_{\tau \wedge t^{\prime}}\right)\right] .
$$

From Lemma 6.2 we know that for $Z_{t}^{\pi}=\left(X_{t}^{\pi}\right)^{\gamma}$

$$
V\left(\tau \wedge t^{\prime}, X_{\tau \wedge t^{\prime}}^{\pi}, p_{\tau \wedge t^{\prime}}\right)=V(t, x, p)+\int_{t}^{\tau \wedge t^{\prime}} Z_{s}^{\pi} \cdot H g\left(s, p_{s}, \pi_{s}\right) d s+\eta_{\tau \wedge t^{\prime}}^{\pi}-\eta_{t}^{\pi} .
$$

Inserting this equation in the preceding inequality yields

$$
0 \geq E^{t, x, p}\left[\int_{t}^{\tau \wedge t^{\prime}} Z_{s}^{\pi} \cdot H g\left(s, p_{s}, \pi_{s}\right) d s\right] .
$$

Let $\tilde{\pi}$ be now a fixed strategy with $\tilde{\pi}_{s} \equiv u \in[0,1]$ for $s \in[t, t+\varepsilon), \varepsilon>0$. Thus, we get

$$
\begin{aligned}
0 & \geq \lim _{t^{\prime} \downarrow t} E^{t, x, p}\left[\frac{1}{t^{\prime}-t} \int_{t}^{\tau \wedge t^{\prime}} Z_{s}^{\tilde{\pi}} \cdot H g\left(s, p_{s}, \tilde{\pi}_{s}\right) d s\right]= \\
& =\lim _{t^{\prime} \downarrow t} E^{t, x, p}\left[\frac{1}{t^{\prime}-t} \int_{t}^{t^{\prime}} Z_{s}^{\tilde{\pi}} \cdot H g\left(s, p_{s}, \tilde{\pi}_{s}\right) d s \mid t^{\prime}<\tau\right] P\left(t^{\prime}<\tau\right)+ \\
& +\lim _{t^{\prime} \downarrow t} E^{t, x, p}\left[\frac{1}{t^{\prime}-t} \int_{t}^{\tau} Z_{s}^{\tilde{\pi}} \cdot H g\left(s, p_{s}, \tilde{\pi}_{s}\right) d s \mid t^{\prime} \geq \tau\right] P\left(t^{\prime} \geq \tau\right)
\end{aligned}
$$

Since $P\left(\tau \leq t^{\prime}\right) \leq 1-e^{-\bar{\lambda}\left(t^{\prime}-t\right)} \rightarrow 0$ for $t^{\prime} \downarrow t$, where $\bar{\lambda}=\max _{k} \lambda_{k}$, we obtain at those points $(t, p)$ where $g$ is differentiable

$$
0 \geq Z_{t}^{\tilde{\pi}} \cdot H g(t, p, u)
$$

From the definition we see that $Z_{t}^{\tilde{\pi}}>0$ which yields $0 \geq H g(t, p, u)$. Let now $(t, p)$ be an arbitrary point, where $g$ might not be differentiable. We know that $\partial g(t, p)=c o\left\{\lim \sup _{n \rightarrow \infty} \nabla g\left(t_{n}, p_{t_{n}}\right), t_{n} \rightarrow t\right\}$ which means by definition that every $\theta \in \partial g(t, p)$ is a convex combination of $\theta^{m}=\limsup _{n \rightarrow \infty} \nabla g\left(t_{n}^{m}, p_{t_{n}^{m}}\right)$ for sequences $t_{n}^{m} \rightarrow t$, along which $g$ is differentiable. Since $g$ is continuous, we obtain

$$
0 \geq L g(t, p ; u)+\frac{1}{\gamma} \theta_{0}^{m}+\frac{1}{\gamma} \sum_{k=1}^{d} \theta_{k}^{m}\left(\sum_{j} q_{j k} p_{j}-p_{k}\left(\lambda_{k}-\hat{\lambda}\right)\right)
$$

which yields the same inequality with $\theta$. Finally since $u$ and $\theta$ are arbitrary, we obtain

$$
0 \geq \sup _{u \in[0,1]}\{L g(t, p ; u)\}+\sup _{\theta \in \partial g(t, p)}\left\{\frac{1}{\gamma} \theta_{0}+\frac{1}{\gamma} \sum_{k=1}^{d} \theta_{k}\left(\sum_{j} q_{j k} p_{j}-p_{k}\left(\lambda_{k}-\hat{\lambda}\right)\right)\right\} .
$$

On the other hand, for $\varepsilon>0$ and $0<t<t^{\prime} \leq T$ with $t^{\prime}-t>0$ small enough there exists a strategy $\pi^{\varepsilon, t^{\prime}} \in \mathcal{U}[t, T]$ with

$$
V(t, x, p)-\varepsilon\left(t^{\prime}-t\right) \leq E^{t, x, p}\left[V\left(\tau \wedge t^{\prime}, X_{\tau \wedge t^{\prime}}^{\pi^{\varepsilon, t^{\prime}}}, p_{\tau \wedge t^{\prime}}\right)\right] .
$$


Again with Lemma 6.2 we obtain

$$
-\varepsilon\left(t^{\prime}-t\right) \leq E^{t, x, p}\left[\int_{t}^{\tau \wedge t^{\prime}} Z_{s}^{\pi^{\varepsilon, t^{\prime}}} \cdot H g\left(s, p_{s}, \pi_{s}^{\varepsilon, t^{\prime}}\right) d s\right] .
$$

Thus, we get

$$
\begin{aligned}
-\varepsilon & \leq E^{t, x, p}\left[\frac{1}{t^{\prime}-t} \int_{t}^{\tau \wedge t^{\prime}} Z_{s}^{\pi^{\varepsilon, t^{\prime}}} \cdot H g\left(s, p_{s}, \tilde{\pi}_{s}^{\varepsilon, t^{\prime}}\right) d s\right] \\
& \leq E^{t, x, p}\left[\frac{1}{t^{\prime}-t} \int_{t}^{\tau \wedge t^{\prime}} Z_{s}^{\pi^{\varepsilon, t^{\prime}}} \cdot \sup _{u \in[0,1]} H g\left(s, p_{s}, u\right) d s\right] .
\end{aligned}
$$

Denote now by $u^{*}(t)$ the maximum point of $u \mapsto L g(t, \phi(t, p), u)$ on $[0,1]$. Since $t \mapsto u^{*}(t)$ is continuous we obtain at those points $(t, p)$ where $g$ is differentiable

$$
-\varepsilon \leq x^{\gamma} \sup _{u \in[0,1]} H g(t, p, u)
$$

and since $\varepsilon>0$ is arbitrary

$$
0 \leq \sup _{u \in[0,1]} H g(t, p, u) .
$$

The analysis if $g$ is not differentiable at $(t, p)$ follows in the same way as before by using the convexity of $\partial g(t, p)$. Altogether it follows that $V$ satisfies the generalized HJB equation.

\section{References}

[1] Benth, F.E., K.H. Karlsen and K. Reikvam (2001): Optimal portfolio selection with consumption and nonlinear integro-differential equations with gradient constraint: a viscosity solution approach, Finance Stoch. 5, 275-303.

[2] Brémaud, P. (1981): Point processes and queues. Springer-Verlag, New York.

[3] Clarke, F.H. (1983): Optimization and nonsmooth analysis. John Wiley \& Sons, New York.

[4] Davis, M. H. A. (1993): Markov models and optimization. Chapman \& Hall, London.

[5] Elliott, R.J., L. Aggoun and J. B. Moore (1994): Hidden Markov models: estimation and control. Springer-Verlag, New York.

[6] Framstad, N.C., B. Øksendal and A. Sulem (1999): "Optimal consumption and portfolio in a jump diffusion market," in: Shiryaev, A. et al. (eds.) Workshop on mathematical finance. Paris: INRIA, 9-20.

[7] Haussmann U.G. and J. Sass (2004): "Optimal terminal wealth under partial information for HMM stock returns," in Mathematics of Finance (Contemp. Math. 351), AMS, Providence, 171-185.

[8] Honda, T. (2003): "Optimal portfolio choice for unobservable and regime-switching mean returns," J. Econ. Dyn. Contr. 28, 45-78. 
[9] Hipp C. and M. Plum (2003): "Optimal investment for investors with state dependent income, and for insurers." Finance Stoch., 7, 299-321.

[10] Kuwana, Y. (1991): "Certainty equivalence and logarithmic utilities in consumption /investment problems." Mathem. Finance, 5, 297-310.

[11] Lakner, P. (1995): "Utility maximization with partial information." Stochastic Process. Appl., 56, 247-273.

[12] Lakner, P. (1998): "Optimal trading strategy for an investor: the case of partial information." Stochastic Process. Appl., 76, 77-97.

[13] Mitronovic, D.S., J. E. Pecaric and A. M. Fink (1993):, Classical and new inequalities in analysis. Kluwer Academic Publishers, Amsterdam.

[14] Müller, A. and D. Stoyan (2002): Comparison methods for stochastic models and risks. Wiley\& Sons, Chichester.

[15] Øksendal, B. and A. Sulem (2005): Applied stochastic control of jump diffusions. SpringerVerlag, Berlin.

[16] Rieder, U. and N. Bäuerle (2005): "Portfolio Optimization with unobservable Markovmodulated drift process," J. Appl. Probab., 42, 362-378.

[17] Rockafellar, R.T. (1970): Convex analysis. Princeton University Press.

[18] Sass, J. and U. G. Haussmann (2004): "Optimizing the terminal wealth under partial information: the drift process as a continuous time Markov chain," Finance Stoch., 8, 553-577.

[19] Schmidli, H. (2002): "On minimizing the ruin probability by investment and reinsurance," Ann. Appl. Probab., 12, 890-907. 